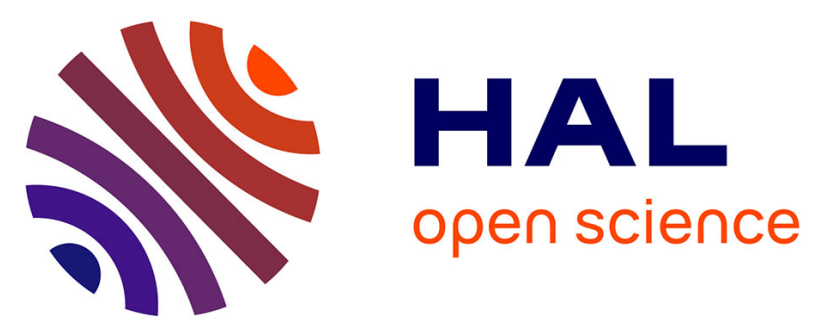

\title{
The implications of previous history of anorexia nervosa in patients with current bulimia nervosa: Alterations in daily functioning, decision-making, and bone status
}

Robertas Strumila, Benédicte Nobile, Laurent Maïmoun, Isabelle Jaussent, Maude Sénèque, Sylvain Thiébaut, Sylvain Iceta, Kathlyne Dupuis-Maurin, Patrick Lefebvre, Philippe Courtet, et al.

\section{To cite this version:}

Robertas Strumila, Benédicte Nobile, Laurent Maïmoun, Isabelle Jaussent, Maude Sénèque, et al.. The implications of previous history of anorexia nervosa in patients with current bulimia nervosa: Alterations in daily functioning, decision-making, and bone status. European Eating Disorders Review, 2019, 10.1002/erv.2712 . hal-02387767

\section{HAL Id: hal-02387767 https://hal.science/hal-02387767}

Submitted on 16 Jul 2020

HAL is a multi-disciplinary open access archive for the deposit and dissemination of scientific research documents, whether they are published or not. The documents may come from teaching and research institutions in France or abroad, or from public or private research centers.
L'archive ouverte pluridisciplinaire HAL, est destinée au dépôt et à la diffusion de documents scientifiques de niveau recherche, publiés ou non, émanant des établissements d'enseignement et de recherche français ou étrangers, des laboratoires publics ou privés. 


\title{
The implications of previous history of anorexia nervosa in patients with current bulimia nervosa: Alterations in daily functioning, decision-making, and bone status
}

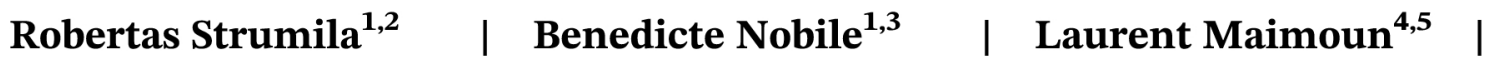

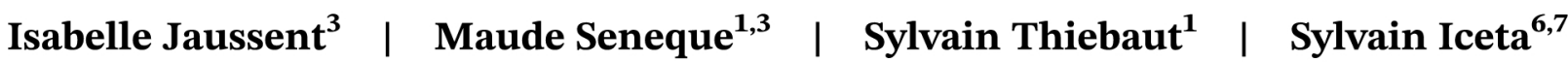 \\ Kathlyne Dupuis-Maurin ${ }^{1}$ ｜ Patrick Lefebvre ${ }^{9}$ | Philippe Courtet ${ }^{1,3}$ | \\ Eric Renard $^{8,9}$ | Sebastien Guillaume S,3 $^{1,3}$
}

${ }^{1}$ Department of Emergency Psychiatry and Post-Acute Care, CHRU Montpellier, France

${ }^{2}$ Psychiatric Clinic, Institute of Clinical Medicine, Faculty of Medicine, Vilnius University, Lithuania

${ }^{3}$ INSERM U1061, Neuropsychiatry: Epidemiological and Clinical Research, University of Montpellier, France

${ }^{4}$ PhyMedExp, Université de Montpellier, INSERM, CNRS, Montpellier, France

${ }^{5}$ Département de Médecine Nucléaire, Hôpital Lapeyronie, Centre Hospitalier Régional Universitaire (CHRU)

Montpellier, 34295, Montpellier, France

${ }^{6}$ Equipe PSYR2, INSERM U1028, CNRS

UMR5292, Université Claude Bernard

Lyon 1, Lyon, France

${ }^{7}$ Referral Center for Eating Disorder, Hospices Civils de Lyon, France

${ }^{8}$ UMR CNRS 5203, INSERM U1191, Institute of Functional Genomics, University of Montpellier, France

${ }^{9}$ Department of Endocrinology, Diabetes, and Nutrition, CHRU Montpellier, France

\section{Correspondence}

Robertas Strumila, MD, Department of Psychiatric Emergency and Acute Care, Hospital Lapeyronie, Avenue Gaston Giraud, 34295 Montpellier CEDEX 5, France.

Email: robertas.strumila@gmail.com

\begin{abstract}
Objective: It is not yet clear what role previous history of anorexia nervosa (AN) plays in the clinical course of bulimia nervosa (BN). We aimed to investigate, using a comprehensive assessment, involving clinical characteristics, daily functioning, cognitive functions, and nutritional and physical markers in $\mathrm{BN}$ patients with a history of $\mathrm{AN}$, and compare them with $\mathrm{BN}$ patients without a history of AN.
\end{abstract}

Methods: Eighty-five patients with a current episode of BN (35 with a lifetime history of $\mathrm{AN}$ ) were analysed. Diagnoses were established according to the DSM-5 criteria. Patients completed neuropsychological tests and filled out validated psychiatric questionnaires. Sociodemographic data and nutritional and somatic illness markers were collected and investigated.

Results: BN patients with a history of previous lifetime AN had worse decision-making ability, worse general and specific functioning, decreased bone density, more antecedent of lifetime suicide attempts, more dietary restraint, and more frequent use of laxatives. The multivariate model shows that the history of AN is closely associated with worse decision-making ability, worse general function, and higher likelihood of lifetime suicide attempts.

Discussion: Prior history of AN is an important clinical question that should receive proper attention when treating $\mathrm{BN}$ patients, as this subgroup of patients may have specific care needs.

\section{K E Y W O R D S}

anorexia nervosa, bone mineral density, bulimia nervosa, decision-making, functioning 


\section{1 | INTRODUCTION}

Bulimia nervosa (BN) is a common eating disorder that has a lifetime incidence rate of 1-3\% in the general population (Smink, van Hoeken, \& Hoek, 2013; Stice, Marti, \& Rohde, 2013). BN, however, is not a homogenous disorder. It has been suggested that $\mathrm{BN}$ can be classified according to the history of AN (Mitchell, Pyle, Eckert, Hatsukami, \& Soll, 1990). Previous studies demonstrated that as much as $50 \%$ of patients with AN develop bulimic symptoms at some point in their lives, and some of them get the clinical diagnosis of BN (Bulik, Sullivan, Fear, \& Pickering, 1997; Eddy et al., 2002; Schaumberg et al., 2019). Moreover, detection of AN patients that are more likely to develop BN could be clinically relevant, as those patients may need particular care (White, 2000).

Efforts to investigate clinical differences between BN patients with and without AN history have shown that patients with a history of AN more often had of all forms of childhood neglect and abuse (Bardone-Cone et al., 2008), lifetime anxiety disorder history (Sullivan, Bulik, Carter, Gendall, \& Joyce, 1996), and were treated for depression and hospitalised (Mitchell et al., 1990). Higher levels of dietary restraint, laxative use, and purging have also been reported in these patients (Bardone-Cone et al., 2008; Mitchell et al., 1990), as well as a lower minimal lifetime (Monteleone, Di Genio, Monteleone, Di Filippo, \& Maj, 2011; White, 2000) and current body mass index (BMI; Bardone-Cone et al., 2008; Sullivan et al., 1996).

Beyond clinical features, one study has investigated neuropsychological functioning in BN patients with a history of AN. The authors concluded that these patients had significantly poorer performance on set-shifting. Meanwhile, decision-making, measured with the Iowa Gambling Task, was significantly worse in all BN patients compared with healthy volunteers; the secondary analysis suggested that only $\mathrm{BN}$ patients with a history of AN had significantly worse performance than healthy volunteers. (Degortes, Tenconi, Santonastaso, \& Favaro, 2016). Finally, one study found lower percentages of body fat and muscle mass, and a higher percentage of extracellular water in patients with former AN (Eddy et al., 2007).

Despite previous efforts to better understand this disorder, the clinical picture of this type of patients remains fragmented-few of the studies investigated beyond clinical characteristics. Almost no studies investigated the nutritional state or somatic features known to be critical on other types of eating disorders such as bone status. Finally, despite the critical relevance of functioning in the burden and prognosis of psychiatric disorders, no studies tried to look at whether these patients have more problems with functioning in their daily lives.

\section{Highlights}

- Prior history of AN in current BN patient predicts worse decision-making abilities.

- Patients with a history of AN had significantly more suicidal attempts in their anamnesis. Therefore, assessment of their current suicide risk should be performed more rigorously.

- Prior history of AN predicts lower general and specific functioning in $\mathrm{BN}$. Therefore, patients may need special care in their communities.

Therefore, the objective of the current study was to do a comprehensive analysis of all these factors coherently, based on DSM-5 criteria, as no previous studies used it. Beyond the clinical characteristics of the disease, we aimed to assess neuropsychological functions (attention, decision-making, executive functioning, and visuospatial abilities) and nutritional (metabolic, somatic, and specifically bone status) parameters of BN patients with a history of $\mathrm{AN}(\mathrm{BN} / \mathrm{AN}+)$ compared with $\mathrm{BN}$ patients without a history of AN (BN/AN-). Finally, particular emphasis was given to general and specific functioning of these patients because of its possible meaningfulness in terms of predicted life quality of the patient.

\section{2 | METHODS}

\section{1 | Participants}

From 2012 to 2014, 85 patients with BN were consecutively recruited, of whom 35 (41\%) had a lifetime history of $\mathrm{AN}$, in a second-line unit specialising in eating disorders (CHU Montpellier, France). The study was conducted according to the Declaration of Helsinki. This ancillary study to a larger project was approved by the local ethics committee (CPP Sud Méditerranée IV), and signed informed consent was obtained from all participants or parents, if the participant was less than 18 years old. Five criteria for inclusion were used: (i) age between 15 and 45 years old, (ii) female gender, (iii) native or fluent in the French language, (iv) diagnosis of $\mathrm{BN}$ according to the DSM-5 criteria, and (v) ability to provide informed consent. Exclusion criteria were (i) inability to understand clinical evaluation and the rules of neuropsychological tests, (ii) getting disability benefits from the government, (iii) pregnancy or lactation, and (iv) deprivation of liberty by judicial or administrative decision. 


\section{2 | Clinical assessment}

The diagnosis of eating disorders was based on the DSM- 5 criteria. Patients were assessed by both a senior psychiatrist and a senior nutritionist experienced in assessment and management of eating disorders, using a nonstructured clinical assessment as well as a structured evaluation with the Mini-International Neuropsychiatric Interview (Version 5.0.0, Lecrubier et al., 1997). Patients also completed the Eating Disorders Examination Questionnaire (EDE-Q; Luce \& Crowther, 1999). In addition, information from medical records and from relatives were collected when available. All diagnosis (current and lifetime) was then established by the consensus of the investigators using the bestestimated procedure using information from all these assessments. In addition, patients filled out the Functioning Assessment Short Test (FAST; Moser et al., 2013; Rosa et al., 2007) questionnaire. The EDE-Q is a 33-item screening tool to evaluate eating disorder symptoms. It measures disordered eating over 28 days and is scored across four subscales that explore the four core clinical dimensions of ED: eating concern, body shape concern, weight concern, and restraint, and total score is calculated by adding all subscales together, and dividing by 4 . Cronbach alpha for EDEQ 4 subscales and total score in this study was .888 . FAST is an interview-administered instrument designed to evaluate functioning across multiple domains in patients with psychiatric disorders. It comprises 24 items divided among six areas of functioning: autonomy, occupational functioning, cognitive functioning, financial issues, interpersonal relationships, and leisure time. Total score is calculated by adding all the subscores together. Cronbach alpha for 6 FAST items and the total score in this study was.781. The higher the overall score, the more severe the difficulties are. In this study, 83 adult patients completed the FAST questionnaire. Therefore, we included all of them in the analyses.

\section{3 | Neuropsychological assessment}

To assess the cognitive functions of the patients, we used four different tests. $\mathrm{d} 2$ test of attention was used to measure sustained attention and concentration. It is a validated, widely used instrument. During it, participants receive a so-called cancellation test, which is composed of 14 lines, each with 47 characters "p" and "d". The characters have one to four dashes that are positioned individually or in pairs above and/or below each letter. The target symbol is a d with two dashes (above or below or both). Thus, a p with one or two dashes and a $d$ with one, three, or four dashes are distracters. A patient has to cross out as many target characters as possible by moving from left to right. (Bates \& Lemay, 2004; Brickenkamp \& Zillmer, 1998). There are many indexes allowing interpretation of this test. The three most used are: $\mathrm{d} 2$ score 1 (or GZ) is the number of characters handled, d2 score 2 (or F) is the number of mistakes (omission or confusion), and the $\mathrm{d} 2$ ratio that is calculated with the formula: $\mathrm{GZ} / \mathrm{F}^{*} 100$ and reflect a global score of this test. The lower the score, the better the attention and concentration of the participants are. In our final analysis, we used the the $\mathrm{d} 2$ ratio.

To measure decision-making, we used the Iowa gambling task (IGT). It is one of the most used tests of this kind. In this game, the participant receives $\$ 2,000$ of game money. Then, during the 100 trials, by taking one of four cards from the deck, the participant has to increase this sum as much as possible. Decks A and B are more profitable in the short term, but less valuable in the long run. Vice versa applies for decks $\mathrm{C}$ and $\mathrm{D}$ - they are more advantageous and safe as the game runs on. After the task, the more money the participant has, the better the result (Bechara, Damasio, Damasio, \& Anderson, 1994). We measured the IGT performance by calculating a global net score (the total number of advantageous selections minus disadvantageous selections). Lower scores represent poor performance, and negative scores indicate a relative preference for the disadvantageous decks.

To evaluate the executive function and mental flexibility, we used "The Brixton spatial anticipation test." It measures the capability to detect changing patterns. A psychologist shows to the participant a series of pages in which there is one filled in a circle and empty circles. In this task, the patient has to predict the position of the filled in circle. The challenge is that the position of filled in circle changes. Then, the total number of errors that were made is converted to a score. The higher the score, the better the mental flexibility and executive functions (Burgess \& Shallice, 1997).

Last, we performed the Rey Osterrieth complex figure test (ROCFT). It assesses visual memory and visuospatial constructional ability. The participant is given the ROCF stimulus card. Then, the study subjects have to reproduce this figure instantly and one more time after $30 \mathrm{~min}$. The score is calculated with a scale from 1 to 4 , where 1 is the best score. The scoring system includes accuracy, organisation, location, and reproduction time (Shin, Park, Park, Seol, \& Kwon, 2006). Because few patients scored 4 on this test, patients who score 3 and 4 were grouped together. 


\section{4 | Resting energy expenditure, areal bone mineral density, and blood assays}

Resting energy expenditure was measured over a period of at least $30 \mathrm{~min}$ by indirect calorimetry (Quark RMR, Cosmed, Rome, Italy) after an overnight fast. Areal bone mineral density (aBMD; grammes per square centimetre) was determined by Dual-energy X-ray absorptiometry (Hologic QDR-4500A, Hologic, Inc., Waltham, MA) at specific bone sites: the anteroposterior lumbar spine (L1L4) and hip. All analyses and scanning were executed by the same operator to ensure consistency after following standard quality control procedures. Quality control for Dual-energy X-ray absorptiometry was checked daily by scanning a lumbar spine phantom consisting of calcium hydroxyapatite embedded in a cube of a thermoplastic resin (DPA/QDR-1; Hologic x-calibre anthropometric spine phantom). The CVs given by the manufacturer were $0.8 \%$ for the spine and $1.1 \%$ at the hip. Blood samples $(25 \mathrm{ml})$ were routinely collected in the morning (8:30-9:30 a.m.) in sterile cool tubes by a standard procedure and analysed in the laboratory using usual assay techniques. Bioimpedance was performed on a Bodystat Quadscan 4000 (BQ4000; Bodystat Ltd., Douglas, UK) to measure fat mass. Measurements were taken in the supine position using two electrodes applied to the right hand and right leg.

\subsection{Statistical analysis}

Categorical variables are presented as percentages, and quantitative variables - as means with standard deviation $(S D)$. Demographic, clinical characteristic, neuropsychological performance, and somatic measurement data between BN patients with AN lifetime history and BN patients without $\mathrm{AN}$ lifetime history were analysed using logistic regression models.

Variables with $p$-value $<.05$ in the univariate analysis were included in a logistic regression analysis. Six independent predictors were chosen: EDE-Q, restriction scores, spine BMD, IGT total scores, FAST total scores, laxatives use, and lifetime suicidal attempts. As one of the limitations of logistic regression analysis is that it can deal with only a limited number of factors when there is a small number of observations (10 or more observations per variable.), two logistic regressions models were performed. One included the four variables of the clinical data and IGT net score, and another included the four variables of the clinical data and BMD. It should be noted that in the multivariate analysis, we did not include several variables into the model that had a $p$-value less than.05. First of them was the lowest lifetime BMI.
Because this variable is consubstantial to the history of AN itself, we decided not to include it, because they are interrelated $(R=.373, p<.001)$. Second, only one variable for from FAST was selected for the same reason (autonomy score and total score $R=.767, p<.001$; interpersonal relationships and total score $R=.892, p<$ .001) - the total score of the FAST questionnaire. Another was the spine and hip osteodensitometry score. All the variables of the data on bones are highly interrelated as well (osteodensitometry score of the hip and spine $R=.741, p<.001$ ) and could not be included in the same multivariate model. Therefore, we included only one of them, which is the most important clinically-the spine osteodensitometry score. (Schorr et al., 2017) The significance level was set at $p \leq .050$. Analyses were performed using the SPSS statistical software (version 23.0.0.2; IBM SPSS Statistics for Windows. Armonk, NY: IBM Corp).

\section{3 | RESULTS}

Clinical characteristics of BN patients with a lifetime history of $\mathrm{AN}(\mathrm{BN} / \mathrm{AN}+)$ and without a history of $\mathrm{AN}$ (BN/AN-) are presented in Table 1. BN/AN+ patients had significantly lower lifetime BMI than BN/AN- ( $p$ value < .002), whereas actual BMI did not differ between the two groups ( $p$-value $=.873)$. Neither age $(p$-value $=$ $.154)$, nor duration of eating disorders ( $p$-value $=.235$ ) were different. The global score of the EDE-Q was not significantly different between the groups ( $p$-value.165). However, BN/AN+ patients had significantly higher scores in restraint subscale ( $p$-value $=.007) . \mathrm{BN} / \mathrm{AN}+$ patients were more likely to use laxatives ( $p$-value = .027). Vomiting and potomania were not different between groups. In terms of psychiatric comorbidity, $\mathrm{BN} / \mathrm{AN}+$ patients were also more likely to use tobacco $(p$-value $=.050)$ and have a lifetime suicide attempt history ( $p$-value $=.048$ ). Comorbid mood, anxiety, and substance abuse disorders were not different between the two groups. There were also no differences in terms of regular menses between the two groups ( $p=.331$ ).

Concerning the association between neuropsychological performance and history of AN (Table 2), there was a trend for an association for $\mathrm{BN} / \mathrm{AN}+$ patients to have worse sustained attention (reflecting by a worse global score to $\mathrm{d} 2$ test; $p=.073$ ). BN/AN+ patients had significantly lower scores on IGT total score $(p$-value $=.076)$. Finally, none of the other neuropsychological performances were significantly different between the two groups.

General and specific functioning of patients is summarised in Table 3. According to the FAST scale, 
T A B LE 1 Association between sociodemographic and clinical characteristics and history of anorexia nervosa

\begin{tabular}{|c|c|c|c|c|c|}
\hline \multirow[b]{4}{*}{ Variables } & \multicolumn{4}{|c|}{ History of anorexia nervosa } & \multirow[b]{4}{*}{$P$-value } \\
\hline & \multicolumn{2}{|c|}{ No } & \multicolumn{2}{|c|}{ Yes } & \\
\hline & \multicolumn{2}{|c|}{$n N=\mathbf{5 0}$} & \multicolumn{2}{|c|}{$N n=35$} & \\
\hline & $\mathbf{n}$ & $\%$ & $\mathbf{n}$ & $\%$ & \\
\hline Age, years ${ }^{(1) a}$ & \multicolumn{2}{|c|}{$26.11(7.49)$} & \multicolumn{2}{|c|}{$28.40(6.84)$} & .154 \\
\hline Study level, years ${ }^{(1) a}$ & \multicolumn{2}{|c|}{$13.17(2.30)$} & \multicolumn{2}{|c|}{$14.20(2.11)$} & .055 \\
\hline Children &.. & .. &.. & .. & .355 \\
\hline No & 41 & 82.0 & 25 & 73.5 & .. \\
\hline Yes & 9 & 18.0 & 9 & 26.5 & .. \\
\hline In couple &.. & .. & .. & .. & .401 \\
\hline No & 38 & 76.0 & 23 & 55.2 & .. \\
\hline Yes & 12 & 24.0 & 11 & 44.8 & .. \\
\hline Working &.. & .. & .. & .. & .566 \\
\hline No & 4 & 8.0 & 4 & 11.8 & .. \\
\hline Yes & 46 & 92.0 & 30 & 88.2 & .. \\
\hline Diseases characteristics &.. & .. & .. & .. & .. \\
\hline Actual BMI, $\mathrm{kg} / \mathrm{m}^{2(1) a}$ & \multicolumn{2}{|c|}{$21.97(2.65)$} & \multicolumn{2}{|c|}{$21.88(2.91)$} & .871 \\
\hline Minimal BMI, $\mathbf{k g} / \mathbf{m}^{2(1) a}$ & \multicolumn{2}{|c|}{$18.67(2.81)$} & \multicolumn{2}{|c|}{$16.54(2.34)$} & .002 \\
\hline Maximal BMI, $\mathrm{kg} / \mathrm{m}^{2(1) a}$ & \multicolumn{2}{|c|}{$24.79(3.08)$} & \multicolumn{2}{|c|}{$25.51(3.73)$} & .355 \\
\hline BN duration, years ${ }^{(1) a}$ & \multicolumn{2}{|c|}{$8.44(7.38)$} & \multicolumn{2}{|c|}{$7.26(7.09)$} & .625 \\
\hline ED duration, years ${ }^{(1) a}$ & \multicolumn{2}{|c|}{$8.44(7.38)$} & \multicolumn{2}{|c|}{$10.98(7.35)$} & .213 \\
\hline Hospitalization for ED & \multicolumn{2}{|l|}{.. } & \multicolumn{2}{|l|}{.. } & .817 \\
\hline No & 32 & 65.3 & 22 & 62.9 & .. \\
\hline Yes & 17 & 34.7 & 13 & 37.1 & .. \\
\hline Regular menstrual cycle & \multicolumn{2}{|l|}{.. } & \multicolumn{2}{|l|}{.. } & .325 \\
\hline No & 14 & 35.0 & 14 & 46.7 & .. \\
\hline Yes & 26 & 65.0 & 16 & 53.3 & .. \\
\hline Vomiting &.. & .. & .. & .. & .358 \\
\hline No & 11 & 22.4 & 11 & 31.4 & .. \\
\hline Yes & 38 & 77.6 & 24 & 68.6 &.. \\
\hline Potomania &.. & .. &.. & .. & .367 \\
\hline No & 32 & 80.0 & 19 & 70.4 & .. \\
\hline Yes & 8 & 20.0 & 8 & 29.6 & .. \\
\hline Laxatives use &.. & .. & .. & .. & .028 \\
\hline No & 41 & 82.0 & 21 & 60.00 & .. \\
\hline Yes & 9 & 18.0 & 14 & 40.00 & .. \\
\hline Diuretics use &.. & .. & .. & .. & 0.17 \\
\hline No & 46 & 95.8 & 27 & 87.1 & .. \\
\hline Yes & 2 & 4.2 & 4 & 12.9 & .. \\
\hline Physical activity &.. & .. & .. & .. & 0.56 \\
\hline No & 15 & 31.9 & 13 & 38.2 & .. \\
\hline Yes & 32 & 68.1 & 21 & 61.8 & .. \\
\hline
\end{tabular}


TABLE 1 (Continued)

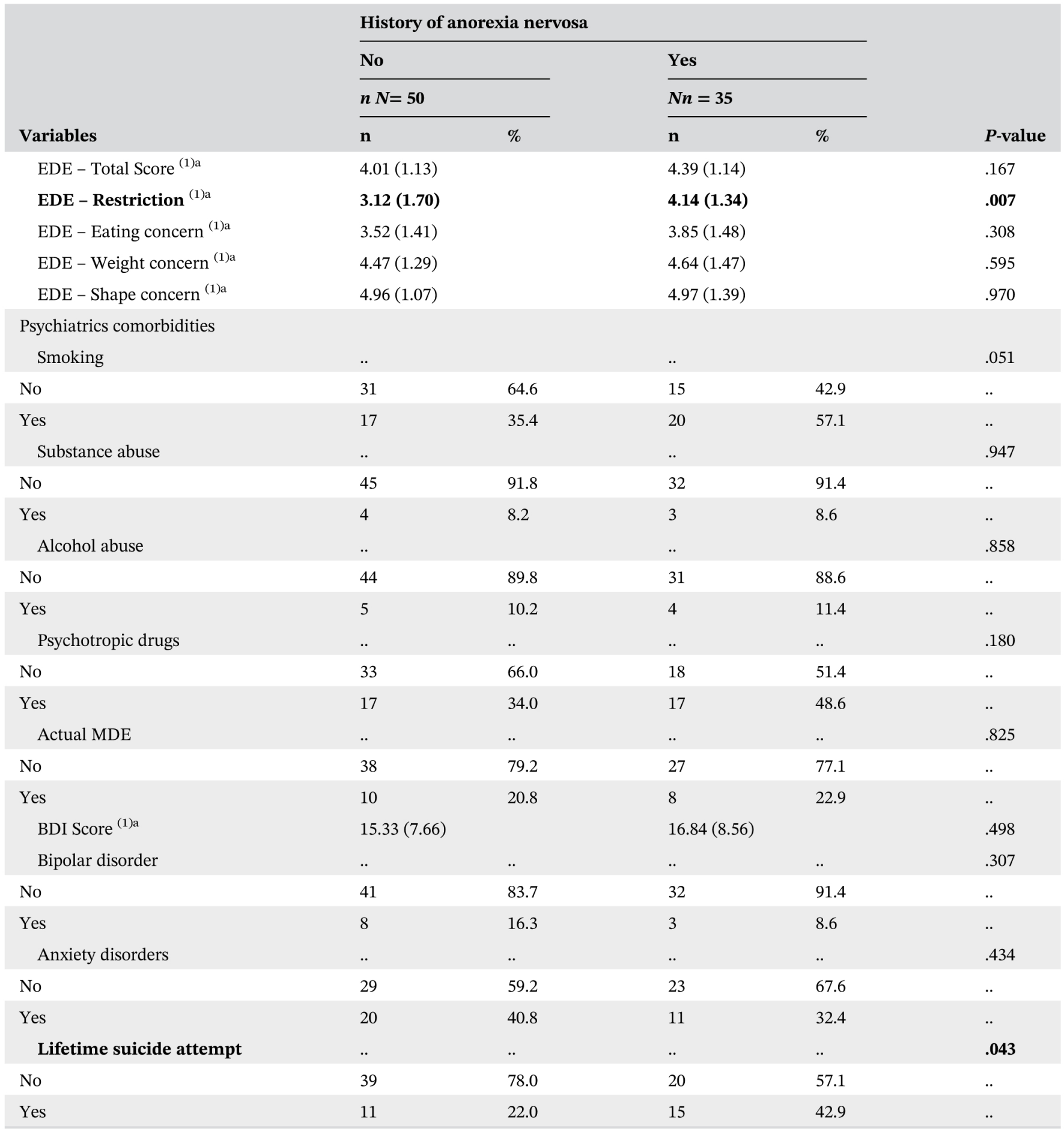

Abbreviations: BDI, beck depression inventory; BMI, - body mass index; ED, - eating disorder; EDE, - eating disorder examination; MDE, - major depression episode; BDI - beck depression inventory. Statistically significant variables and values are bolded.

Continuous variables are expressed as means [(standard deviation (SD)].)

$\mathrm{BN} / \mathrm{AN}+$ patients had higher total scores ( $p$-value $=$ $.025)$, autonomy scores ( $p$-value $=.008)$, and relationship scores $(p$-value $=.033)$, showing worse functioning.

Patients with the history of AN had worse osteodensitometry scores (Table 4) than those without it. Osteodensitometry $p$-value for the spine score was.028 and.014 for the hip. Spine $\mathrm{Z}$ score was significantly lower in the BN/AN+ group $(p=.014)$, and there was a trend for a lower $z$ score of the hip as well $(p=.076)$.

There was no difference between $\mathrm{BN} / \mathrm{AN}+$ and BN/AN- patients to have a slower metabolism as 
T A B L E 2 Association between nNeuropsychological performance and history of aAnorexia nNervosa

\begin{tabular}{|c|c|c|c|c|c|}
\hline \multirow[b]{3}{*}{ Variables } & \multicolumn{4}{|c|}{ History of aAnorexia nNervosa } & \multirow[b]{3}{*}{$P$-value } \\
\hline & \multicolumn{2}{|l|}{ No } & \multicolumn{2}{|l|}{ Yes } & \\
\hline & $\mathbf{n}$ & $\%$ & $\mathbf{n}$ & $\%$ & \\
\hline dD2 Ratio (1)a & $4.45(3.23)$ & & $6.59(6.71)$ & & .073 \\
\hline IGT Total Score ${ }^{(1) a}$ & $21.39(30.90)$ & & $3.22(30.51)$ & & .027 \\
\hline Figure de Rey - style of coping & .. & .. & .. & .. & .619 \\
\hline 1 & 29 & 59.2 & 16 & 50.0 & .. \\
\hline 2 & 12 & 24.5 & 11 & 34.4 & .. \\
\hline$\geq 3$ & 8 & 16.3 & 5 & 15.6 & .. \\
\hline
\end{tabular}

Abbreviation: IGT, - Iowa Gambling Task. Statistically significant variables and values are bolded.

${ }^{a}$ Ccontinuous variables are expressed as means ( $S D$, standard deviation).

${ }^{\mathrm{b}}$ Some neuropsychological tests had missing data. $N n$ for those, that had missing data is reported here: $\mathrm{dD} 2$ ratio $N n=82$; IGT $N n=66$; Brixton $n$ N= 82; FDR $n \mathrm{~N}=81$.

T A B L E 3 General and specific functioning in bulimia patients with a history of anorexia nervosa

\begin{tabular}{|c|c|c|c|}
\hline \multirow[b]{4}{*}{ Variables } & \multicolumn{2}{|c|}{$\begin{array}{l}\text { History of Anorexia } \\
\text { Nervosa }\end{array}$} & \multirow[b]{4}{*}{$P$-value } \\
\hline & \multirow{3}{*}{$\begin{array}{l}\text { No } \\
\frac{\text { Nn }=50}{\operatorname{Mean}(S D)}\end{array}$} & \multirow{3}{*}{$\frac{\text { Yes }}{\frac{\mathrm{n} N=33}{\operatorname{Mean}(S D)}}$} & \\
\hline & & & \\
\hline & & & \\
\hline $\begin{array}{l}\text { FAST - } \\
\text { Autonomy }\end{array}$ & $2.62(2.75)$ & $4.39(2.67)$ & .008 \\
\hline $\begin{array}{l}\text { FAST - } \\
\text { Professional } \\
\text { activity }\end{array}$ & $3.33(4.08)$ & $4.29(4.74)$ & .357 \\
\hline FAST - Cognitive & $5.04(3.88)$ & $5.82(3.78)$ & .360 \\
\hline FAST - Finances & $2.58(2.10)$ & $2.56(2.17)$ & .971 \\
\hline $\begin{array}{l}\text { FAST - } \\
\quad \text { Relationships }\end{array}$ & $6.65(4.79)$ & $9.00(4.45)$ & .033 \\
\hline FAST - Hobbies & $1.80(1.87)$ & $2.09(1.93)$ & .490 \\
\hline $\begin{array}{l}\text { FAST - Total } \\
\text { Score }\end{array}$ & $21.00(14.47)$ & 29.04 (13.98) & .034 \\
\hline
\end{tabular}

Abbreviations: FAST, - functional assessment short test; $S D$, standard deviation. Statistically significant variables and values are bolded.

measured by calorimetry $[1,292.61$ (SD 168.74) vs. 1,360.81 (SD 207.37)], $p$-value.127.

The multivariate model shows that IGT net scores, FAST total scores, and, in one model, lifetime history of suicide attempts were significantly and independently associated with a lifetime diagnosis of AN. (Tables 5 and 6)

\section{DISCUSSION}

The goal of this study was to investigate the clinical significance of a prior history of AN to the course of current BN. Our result adds a significant contribution to the findings of previous studies, suggesting that AN history is an important clinical message for the care of the patient with BN.

In the present study, BN/AN+ patients were more likely to have a lifetime history suicide attempt, use tobacco and laxatives, have a lower minimal lifetime BMI, and have more dietary restraint as measured by EDE-Q. More prevalent laxative use has already been reported in 1990 (Mitchell et al., 1990), as well as more vomiting behaviour, which was not found in our study. Minimal lifetime BMI is well-documented finding, reported by several other studies (Monteleone et al., 2011; White, 2000). Bardone-Cone reported that BN patients with AN history more often had a dietary restriction, which was also found in our sample (Bardone-Cone et al., 2008). However, we did not find a higher likelihood for depression like Mitchel et al. (Mitchell et al., 1990), or higher chance of a comorbid anxiety disorder like Sullivan et al. (Sullivan et al., 1996). Instead, BN patients with AN history had a higher likelihood of lifetime suicide attempt in the present study, which remained significant even in one of the multivariate models. This is an interesting finding because AN patients were more likely to have made a serious suicide attempt whereas BN patients did not differ from the control group according to suicide attempts in a previous study (Guillaume et al., 2011).

In terms of cognitive functions, we investigated attention, executive function, decision-making, and 
T A B L E 4 Association between somatic data and history of aAnorexia nNervosa

\section{History of Anorexia Nervosa}

\begin{tabular}{lllll}
\hline No & & Yes & \\
\hline n & n & & P & -value
\end{tabular}

\section{Variables}

Hematology

Hemoglobin, g/dlL ${ }^{(1) a}$

Albumin, g/lL ${ }^{(1) a}$

Ferritin, $\mu \mathrm{g} / \mathrm{lL}^{(1) \mathrm{a}}$

Vitamin B12, pg/mlL ${ }^{(1) a}$

Intra erythrocyte folate, $\mathrm{ng} / \mathrm{mlL}^{(1) a}$

Inflammation

CRP, mg/lL ${ }^{(1) a}$

Fibrinogen, g/lL ${ }^{(1) a}$

Renal function

Uremia, mmol/1L ${ }^{(1) a}$

Creatinine, $\mu \mathrm{mol} / \mathrm{lL}^{(1) \mathrm{a}}$

Hepatic function

ASAT, UI/lL ${ }^{(1) a}$

ALAT, UI/lL ${ }^{(1) a}$

GGT, UI/1L ${ }^{(1) a}$

Bone Metabolism

ALP, UI/1L ${ }^{(1) a}$

57.04 (16.93)

$2.38(0.08)$

$1.11(0.19)$

Phosphoremia, mmol/lL ${ }^{(1) a}$

Bone Densitometry

Hip BMD (1)a

Spine BMD ${ }^{(1) a}$

Hip Z-score

$\leq-1$

$>--1$

$0.92(0.12)$

$1.01(0.10)$

$0.84(0.14)$

.014

$0.94(0.14)$

$28(80.0)$

$10(41.7)$

14 (58.3)

Spine $Z$ - score

$>--1$

34 (81.0)

8 (19.0)

$\leq-1$

Calorimetry

Basal calorimetry, calories ${ }^{\text {(1)a }}$

Metabolic gap ${ }^{(1) a}$

Fat mass, ${ }^{(1) a}$
1360.81 (207.37)

-3.77 (13.06)

31.38 (9.69)
.378

.687

.953

.534

.510

.768

996

.965

.848

.905

.390

.591

.194

14

076

012

16 (57.1)

12 (42.9)

1292.61 (168.74)

.127

-3.47 (10.85)

Note. Some biological variables had missing data. $n$ for those, that had missing data it is reported here: Hemoglobin' $n=84 ;$ Albumin' $n=77 ;$ Ferritin' $n=77$ Intra erythrocyte folate: $n=81$; CRP: $n=84$; Fibrinogen: $n=63$; Creatinine: $n=81$; ASAT: $n=84$; ALAT: $n=84$; GGT: $n=81$; ALP: $n=81$; Calcium: $n=$ 84; Phosphoremia $n=82$; Bone Densitometry $n=72$, calorimetry: $n=81$. Statistically significant variables and values are bolded.

Continuous variables are expressed as means (SD)

Abbreviations: CRP - c reactive protein; ASAT _ aspartate aminotransferase; ALAT _ , alanine transaminase; ALP, alkaline phosphatase; ASAT, aspartate aminotransferase; BMD, bone mineral density; CRP, c-reactive protein; GGT,- gamma glutamyltransferase.; ALP - alkaline phosphatase: BMD - bone mineral density

Some biological variables had missing data. $\mathrm{N}$ for those, that had missing data it is reported here: Hemoglobin $-\mathrm{N}=84 ;$ Albumin $-\mathrm{N}=77$; Ferritin $-\mathrm{N}=77$; Intra erythrocyte folate: $\mathrm{N}=81$; CRP: $\mathrm{N}=84$; Fibrinogen: $\mathrm{N}=63$; Creatinine: $\mathrm{N}=81$; ASAT: $\mathrm{N}=84$; ALAT: $\mathrm{N}=84$; GGT: N=81; ALP: N=81; Calcium: N=84; Phosphoremia $\mathrm{N}=82$; Bone Densitometry $\mathrm{N}=72$, calorimetry: $\mathrm{N}=81^{\mathrm{a}}$ Continuous variables are expressed as means (SD). 
TA B LE 5 Multivariate analysis of the associations between clinical and neuropsychological characteristics and history of AN (22 patients with history of AN vs. 33 patient without history of AN)

\begin{tabular}{llc} 
Variable & OR [95\% CI] & $P$-value \\
\hline Laxatives use & & .524 \\
\hline Yes & $1.87[0.27, ; 12.9]$ & .217 \\
\hline EDE - Restriction & $1.42[0.81, ; 2.47]$ & \\
\hline $\begin{array}{l}\text { Lifetime suicide } \\
\quad \text { attempt }\end{array}$ & & .054 \\
\hline Yes & $7.47[0.97, ; 57.7]$ & $\mathbf{. 0 2 7}$ \\
\hline IGT Total Score & $0.97[0.94, ; 1.00]$ & $\mathbf{0 1 8}$ \\
\hline FAST - Total Score & $1.09[1.01, ; 1.16]$ & \\
\hline
\end{tabular}

Abbreviations: AN, anorexia nervosa; CI, confidence interval; FAST, functional assessment short test; EDE, eating disorder examination; IGT, Iowa Gambling Task; OR, odds ratio. Statistically significant variables and values are bolded.

TA B LE 6 Multivariate analysis of the associations between clinical and somatic characteristics and history of AN (24 patients with history of AN vs. 35 patient without history of AN)

\begin{tabular}{lcc} 
Variable & OR $[\mathbf{9 5 \%} \mathbf{C I}]$ & $\boldsymbol{P}$-value \\
Laxatives use & & .452 \\
Yes & $1.77[0.40, ; 7.84]$ & .354 \\
EDE - Restriction & $1.26[0.78, ; 2.03]$ & \\
Lifetime suicide attempt & & $\mathbf{0 3 6}$ \\
Yes & $6.24[1.13, ; 34.4]$ & .150 \\
Spine BMD & $0.01[0.00, ; 4.77]$ & $\mathbf{. 0 4 9}$ \\
FAST - Total sScore & $1.05[1.00, ; 1.10]$ & \\
\hline
\end{tabular}

Abbreviations: AN, anorexia nervosa; BMD, bone mineral density; CI, confidence interval; FAST, functional assessment short test; EDE, eating disorder examination; OR, odds ratio. Statistically significant variables and values are bolded.

visuospatial abilities. One of the most studied cognitive functions in eating disorders patients is decision-making. It is well documented that decision-making is impaired in these patients, as they tend to exhibit behaviours resulting in fast gratification instead of delaying it for later, but with a better outcome (Bodell et al., 2014; Guillaume et al., 2015). This finding is robust, replicated, and was even confirmed in male patients with AN. (Tchanturia et al., 2012). However, there are almost no studies investigating whether there is a difference in decision-making between BN/AN+ versus BN/ANpatients. One existing study by Degortes et al., which used the IGT, found that decision-making was impaired in all bulimia patients compared with the healthy women. Subgroup analysis suggests worse decisionmaking performance in BN patient with the previous history of AN compared with control, whereas "pure BN" was in-between and nondifferent of the two other groups (Degortes et al., 2016). In the present study, we confirm and extend these findings, demonstrating that previous history of AN in BN patients was significantly associated with worse decision-making abilities, measured by the
IGT, compared with "pure" BN patients. This association remained significant even in the more robust multivariate logistic regression model. It has been previously suggested that another pathway underlines the impairment in IGT in BN than in AN (Chan et al., 2014). This also seems true with other dimensions of decision-making, such as delay discounting (Steward et al., 2017). These findings suggest that the way to think about these decision-making impairments and address them are different in AN and BN patients. Nevertheless, these studies were not taking into account previous diagnosis (i.e.: former $\mathrm{AN}$ in $\mathrm{BN}$ group or former BN in AN group). Therefore, it is interesting which mechanism underlies impaired decision-making in our BN/AN+ patients sample whether it is more similar to the mechanism in $\mathrm{BN}$ or AN. Determination of the mechanisms was impossible in our study, but, we suggest that future studies that assess decision-making abilities in eating disorders patients should take into account the lifetime trajectory of eating disorders and not only the current diagnosis. By contrast and in accordance with the study by Degortes et al. (Degortes et al., 2016), central coherence seems not to be 
associated with AN history. Finally, our results suggest no difference in term of set-shifting between the two groups. This is partially contradictory with the study of Degortes et al. This discrepancy might be explained by methodological difference (the task used were different Brixton versus Wisconsin) and should be further explored in future studies.

Attention is another essential cognitive function that has been shown to be impaired in eating disorders patients. The study by Seitz et al. using an functional MRI (Seitz et al., 2016) found dysfunction of attention network in BN patients, associated with overactivation of parietooccipital regions and reduced deactivation of the default mode network. Also, the difference in attention bias has been demonstrated in restrictive and purging type of AN (Enoch-Levy et al., 2018). In our study, there was a tendency for BN patients with a history of AN to have worse attention $(p=.07)$. It is, therefore, an intriguing hypothesis for further studies with bigger sample sizes to investigate whether a history of AN would predict worse attention in $\mathrm{BN}$ patients.

Interesting findings emerged from looking at the general and specific functioning of BN/AN+ versus BN/ANpatients. To our knowledge, this is the first study to analyse whether there are differences in general and specific functioning between bulimia patients with a history of anorexia. We have found statistically significantly impaired general functioning, which remained significant in the multivariate model. This finding could be helpful to caring clinicians because by asking about whether a patient with bulimia had an anorexic episode, it could give valuable clinical information and help to make better decisions for care. However, we could not conclude whether this is explicitly related to the history of anorexia nervosa itself. Prospective studies would be needed, which assessed functioning in anorexia patients and then follows them, to see who develops bulimia and whether they retain impairment in functioning.

In terms of specific functioning domains, two categories were significantly more impaired-relationships and autonomy. Because they are less able to live an independent life, and it is also harder to have meaningful relationships, these patients may need extra social care in their communities. Our results do not determine whether these difficulties result from the former anorexia, and the literature on functioning in all eating disorders patients is scarce. The study by Moser et al. (Moser et al., 2013) investigated differences between psychosocial functioning in patients with different types of eating disorders. They specifically looked at patients with restrictive subtype and purging subtype of eating disorder. It was found that there were no differences in total FAST scores or specific domains. Wentz et al. followed adolescents with anorexia nervosa for 10 years. They concluded that according to the GAF scale, half of the patients were of poor functioning. (Wentz, Gillberg, Gillberg, \& Rastam, 2001).

Regarding nutritional biomarkers, there were no significant differences found. This suggests that when patients remit from AN and starvation state, even if they develop $\mathrm{BN}$, their nutritional state recovers without any apparent insufficiencies, which can be reassuring for both the patient and the physician. On the contrary, some interesting findings emerged when bone status was analysed. BN/AN+ patients more often had decreases on bone mass density on all sites, and a significant decrease in both the spine and hip osteodensitometry scores and Spine Z score, indicating that prior history of AN is associated with decreased aBMD. Bone metabolism alterations are very well documented in AN (Fazeli \& Klibanski, 2014; Maïmoun et al., 2018) and cause health problems, like fractures, for these patients. Even though there are also some studies that bone metabolism is altered in BN (Robinson, Micali, \& Misra, 2017), usually, it is a less pronounced problem. National Institute for Health and Care Excellence guidelines recommend a aBMD scan in AN patients, but not in BN (National Institute for Health and Care Excellence., 2017). Because the association between bone loss and previous history of AN lost significance in multivariate analysis, these data call for more studies with bigger sample sizes and suggests that it could be important in the clinic to also assess aBMD in BN/AN+ patients.

This study has some strengths. First of all, a comprehensive assessment was performed, whereas previous studies were more focused on a specific aspect of the eating disorder. All patients were recruited according to the strict procedure, and the diagnosis was confirmed in tertiary eating disorders centre. We used validated and wellknown tools for evaluations. In this study, we confirmed findings by other authors like more dietary restraint, more use of laxatives, and a lower lifetime BMI of BN/AN+ patients. This is important because the literature on this subject is very limited. Our study is also the first that presented some new findings in this subgroup of patients, namely impaired decision-making, higher likelihood of lifetime suicide attempt history, and impaired general and specific functioning. Also, we showed that these patients have significantly lower bone mass and a tendency for lower metabolic rate. This is valuable information, as it shows that $\mathrm{BN}$ patients with a previous history of AN have a higher need for careful attention to nutrition.

There are some limitations as well. First of all, our study sample was small, which may limit the generalizability of our results. A study with a larger sample size of BN patients with or without previous history of AN is 
warranted. It would be optimal if that study would have a healthy control group because we did not have that group in our sample. Another important aspect would be to control in detail for medication and drug use. Because some medications have neuroprotective effects (i.e., antidepressants, and antipsychotics), others could have neurodamaging effects (benzodiazepines, and cannabis). Therefore, it is interesting to see how the use of medicines may modulate the clinical outcomes. Regarding statistical analysis, one limitation of this study was that we were not able to perform a unique logistic regression model due to only a partial overlap on IGT and osteodensitometry assessment. Therefore, we performed two models, to increase the statistical power of each. Another limitation is that we studied only those patients who were admitted to our second-line unit. Hence, our results are generalizable only to patients admitted to a specialised unit.

\section{5 | CONCLUSION}

This study aimed to do a comprehensive analysis of neuropsychological tests, general and specific functioning, and metabolic and somatic parameters of $\mathrm{BN}$ patients with a previous history of AN. Besides the previously known specific features such as more restriction, laxative use, lower lifetime BMI, and tobacco use, these patients also have worse decision-making abilities and general and specific functioning, which is most likely residual impairment from AN, therefore needs special management. Patients also had a higher likelihood of having a prior suicide attempt, which suggests that these patients require a more rigorous assessment of their current suicide risk. It is therefore important to screen for the previous history of AN in daily practice when caring for $\mathrm{BN}$ patients.

\section{ACKNOWLEDGEMENTS}

None.

\section{REFERENCES}

Bardone-Cone, A. M., Maldonado, C. R., Crosby, R. D., Mitchell, J. E., Wonderlich, S. A., Joiner, T. E., ... le Grange, D. (2008). Revisiting differences in individuals with bulimia nervosa with and without a history of anorexia nervosa: Eating pathology, personality, and maltreatment. The International Journal of Eating Disorders, 41(8), 697-704. https://doi.org/10. 1002/eat.20567

Bates, M. E., \& Lemay, E. P. (2004). The d2 Test of attention: construct validity and extensions in scoring techniques. Journal of the International Neuropsychological Society: JINS, 10(3), 392-400. https://doi.org/10.1017/S135561770410307X

Bechara, A., Damasio, A. R., Damasio, H., \& Anderson, S. W. (1994). Insensitivity to future consequences following damage to human prefrontal cortex. Cognition, 50(1-3), 7-15. https://doi.org/8039375, https://doi.org/10.1016/0010-0277(94) 90018-3

Bodell, L. P., Keel, P. K., Brumm, M. C., Akubuiro, A., Caballero, J., Tranel, D., ... McCormick, L. M. (2014). Longitudinal examination of decision-making performance in anorexia nervosa: before and after weight restoration. Journal of Psychiatric Research, 56, 150-157. https://doi.org/10.1016/j.jpsychires.2014. 05.015

Brickenkamp, R., \& Zillmer, E. (1998). The d2 Test of Attention. Seattle, Washington: Hogrefe \& Huber Publishers.

Bulik, C. M., Sullivan, P. F., Fear, J., \& Pickering, A. (1997). Predictors of the development of bulimia nervosa in women with anorexia nervosa. The Journal of Nervous And Mental Disease, 185(11), 704-707. Retrieved from. http://www.ncbi.nlm.nih. gov/pubmed/9368548

Burgess, T. S., \& Shallice, T. (1997). The Hayling and Brixton Tests. Bury St Edmunds: Thames Valley Test Company.

Chan, T. W. S., Ahn, W. Y., Bates, J. E., Busemeyer, J. R., Guillaume, S., Redgrave, G. W., ... Courtet, P. (2014). Differential impairments underlying decision making in anorexia nervosa and bulimia nervosa: a cognitive modeling analysis. International Journal of Eating Disorders, 47(2), 157-167. https://doi.org/10.1002/eat.22223

Degortes, D., Tenconi, E., Santonastaso, P., \& Favaro, A. (2016). Executive functioning and visuospatial abilities in bulimia nervosa with or without a previous history of anorexia Nervosa. European Eating Disorders Review: The Journal of the Eating Disorders Association, 24(2), 139-146. https://doi.org/10.1002/ erv. 2430

Eddy, K. T., Dorer, D. J., Franko, D. L., Tahilani, K., ThompsonBrenner, H., \& Herzog, D. B. (2007). Should bulimia nervosa be subtyped by historyof anorexia nervosa? A longitudinal validation. International Journal of Eating Disorders, 40(S3), S67-S71. https://doi.org/10.1002/eat.20422

Eddy, K. T., Keel, P. K., Dorer, D. J., Delinsky, S. S., Franko, D. L., \& Herzog, D. B. (2002). Longitudinal comparison of anorexia nervosa subtypes. The International Journal of Eating Disorders, 31(2), 191-201. Retrieved from. http://www.ncbi.nlm.nih. gov/pubmed/11920980

Enoch-Levy, A., Bar-Haim, Y., Hamdan, S., Treasure, J., DubnovRaz, G., Stein, D., ... Gilon Mann, T. (2018). Different attention bias patterns in anorexia nervosa restricting and binge/purge types. European Eating Disorders Review, 26(4), 293-301. https://doi.org/10.1002/erv.2593

Fazeli, P. K., \& Klibanski, A. (2014). Anorexia nervosa and bone metabolism. Bone, 66, 39-45. https://doi.org/10.1016/j.bone. 2014.05.014

Guillaume, S., Gorwood, P., Jollant, F., Van den Eynde, F., Courtet, P., \& Richard-Devantoy, S. (2015). Impaired decision- 
making in symptomatic anorexia and bulimia nervosa patients: a meta-analysis. Psychological Medicine, 45(16), 3377-3391. https://doi.org/10.1017/S003329171500152X

Guillaume, S., Jaussent, I., Olié, E., Genty, C., Bringer, J., Courtet, P., \& Schmidt, U. (2011). Characteristics of suicide attempts in anorexia and bulimia nervosa: a case-control study. PLoS ONE, 6(8), e23578. https://doi.org/10.1371/journal.pone. 0023578

Lecrubier, Y., Sheehan, D. V., Weiller, E., Amorim, P., Bonora, I., Sheehan, K. H., ... Dunbar, G. C. (1997). The Mini International Neuropsychiatric Interview (MINI). A short diagnostic structured interview: Reliability and validity according to the CIDI. European Psychiatry, 12(5), 224-231. https://doi.org/10.1016/ S0924-9338(97)83296-8

Luce, K. H., \& Crowther, J. H. (1999). The reliability of the Eating Disorder Examination-Self-Report Questionnaire Version (EDE-Q). The International Journal of Eating Disorders, 25(3), 349-351. Retrieved from. http://www.ncbi.nlm.nih.gov/ pubmed/10192002

Maïmoun, L., Guillaume, S., Lefebvre, P., Bertet, H., Seneque, M., Philibert, P., ... Sultan, C. (2018). Effects of the two types of anorexia nervosa (binge eating/purging and restrictive) on bone metabolism in female patients. Clinical Endocrinology, 88(6), 863-872. https://doi.org/10.1111/cen.13610

Mitchell, J. E., Pyle, R. L., Eckert, E. D., Hatsukami, D., \& Soll, E. (1990). Bulimia nervosa with and without a history of anorexia nervosa. Comprehensive Psychiatry, 31(2), 171-175. Retrieved from. http://www.ncbi.nlm.nih.gov/pubmed/2311384

Monteleone, P., Di Genio, M., Monteleone, A. M., Di Filippo, C., \& Maj, M. (2011). Investigation of factors associated to crossover from anorexia nervosa restricting type (ANR) and anorexia nervosa binge-purging type (ANBP) to bulimia nervosa and comparison of bulimia nervosa patients with or without previous ANR or ANBP. Comprehensive Psychiatry, 52(1), 56-62. https://doi.org/10.1016/j.comppsych.2010.05.002

Moser, C. M., Lobato, M. I. R., Rosa, A. R., Thomé, E., Ribar, J., Primo, L., ... Brunstein, M. G. (2013). Impairment in psychosocial functioning in patients with different subtypes of eating disorders. Trends in Psychiatry and Psychotherapy, 35 (2), 111-118. https://doi.org/10.1590/S2237-60892013000200004 National Institute for Health and Care Excellence. (2017). Eating disorders: recognition and treatment. National Institute for Health and Care Excellence, (May). Retrieved from https:// www.nice.org.uk/guidance/ng69/chapter/

Recommendations\#treating-anorexia-nervosa

Robinson, L., Micali, N., \& Misra, M. (2017). Eating disorders and bone metabolism in women. Current Opinion in Pediatrics, 29 (4), 488-496. https://doi.org/10.1097/MOP.0000000000000508

Rosa, A. R., Sánchez-Moreno, J., Martínez-Aran, A., Salamero, M., Torrent, C., Reinares, M., ... Vieta, E. (2007). Validity and reliability of the Functioning Assessment Short Test (FAST) in bipolar disorder. Clinical Practice and Epidemiology in Mental Health, 3(1), 5. https://doi.org/10.1186/1745-0179-3-5

Schaumberg, K., Jangmo, A., Thornton, L. M., Birgegård, A., Almqvist, C., Norring, C., ... Bulik, C. M. (2019). Patterns of diagnostic transition in eating disorders: a longitudinal population study in Sweden. Psychological Medicine, 49(5), 819-827. https://doi.org/10.1017/S0033291718001472

Schorr, M., Thomas, J. J., Eddy, K. T., Dichtel, L. E., Lawson, E. A., Meenaghan, E., ... Miller, K. K. (2017). Bone density, body composition, and psychopathology of anorexia nervosa spectrum disorders in DSM-IV vs DSM-5. The International Journal of Eating Disorders, 50(4), 343-351. https://doi.org/10.1002/eat. 22603

Seitz, J., Hueck, M., Dahmen, B., Schulte-Rüther, M., Legenbauer, T., Herpertz-Dahlmann, B., \& Konrad, K. (2016). Attention network dysfunction in bulimia nervosa-an fMRI study. PLOS ONE, 11(9), e0161329. https://doi.org/10.1371/ journal.pone.0161329

Shin, M.-S., Park, S.-Y., Park, S.-R., Seol, S.-H., \& Kwon, J. S. (2006). Clinical and empirical applications of the ReyOsterrieth Complex Figure Test. Nature Protocols, 1(2), 892-899. https://doi.org/10.1038/nprot.2006.115

Smink, F. R. E., van Hoeken, D., \& Hoek, H. W. (2013). Epidemiology, course, and outcome of eating disorders. Current Opinion in Psychiatry, 26(6), 543-548. https://doi.org/10.1097/YCO. 0b013e328365a24f

Steward, T., Mestre-Bach, G., Vintró-Alcaraz, C., Agüera, Z., Jiménez-Murcia, S., Granero, R., \& Fernández-Aranda, F. (2017). Delay discounting of reward and impulsivity in eating disorders: from anorexia nervosa to binge eating disorder. European Eating Disorders Review, 25(6), 601-606. https://doi. org/10.1002/erv.2543

Stice, E., Marti, C. N., \& Rohde, P. (2013). Prevalence, incidence, impairment, and course of the proposed DSM-5 eating disorder diagnoses in an 8-year prospective community study of young women. Journal of Abnormal Psychology, 122(2), 445-457. https://doi.org/10.1037/a0030679

Sullivan, P. F., Bulik, C. M., Carter, F. A., Gendall, K. A., \& Joyce, P. R. (1996). The significance of a prior history of anorexia in bulimia nervosa. The International Journal of Eating Disorders, 20(3), 253-261. DOI: https://doi.org/10.1002/( SICI)1098-108X(199611)20:3<253::AID-EAT4>3.0.CO;2-N

Tchanturia, K., Liao, P.-C., Forcano, L., Fernández-Aranda, F., Uher, R., Treasure, J., ... Campbell, I. C. (2012). Poor decision making in male patients with anorexia nervosa. European Eating Disorders Review, 20(2), 169-173. https://doi.org/10.1002/ erv. 1154

Wentz, E., Gillberg, C., Gillberg, I. C., \& Rastam, M. (2001). Tenyear Follow-up of Adolescent-onset anorexia nervosa: psychiatric disorders and overall functioning scales. Journal of Child Psychology and Psychiatry, 42(5), 613-622. https://doi.org/10. 1111/1469-7610.00757

White, J. H. (2000). Symptom development in bulimia nervosa: a comparison of women with and without a history of anorexia nervosa. Archives of Psychiatric Nursing, 14(2), 81-92. Retrieved from. http://www.ncbi.nlm.nih.gov/pubmed/10783526 